УДК $517.55+517.96$

\title{
The Cauchy Problem for Multidimensional Difference Equations and the Preservation of the Hierarchy of Generating Functions of its Solutions
}

\author{
Evgeny K. Leinartas* \\ Tatiana I. Yakovleva ${ }^{\dagger}$ \\ Institute of Mathematics and Computer Science \\ Siberian Federal University \\ Svobodny, 79, Krasnoyarsk, 660041
}

Russia

Received 15.06.2018, received in revised form 20.08.2018, accepted 20.09.2018

We study the dependence of the properties of the generating function of the solution of the Cauchy problem on the properties of the generating function of the initial data for a difference equation with constant coefficients in a rational point cone. Conditions are found under which the generating functions of the solution remain in the same classes as the generating functions of the initial data.

Keywords: multidimensional difference equations, Cauchy problem, hierarchy of generating function, Hadamard composition.

DOI: $10.17516 / 1997-1397-2018-11-6-712-722$

\section{Introduction}

Difference equations are an important tool for investigating combinatorial problems. One way to describe the solution space of a multidimensional difference equation is to specify additional conditions («boundary» or «initial data») for a solution whose fulfillment ensures its existence and uniqueness (the Cauchy problem) (see, for example, [1,2]). The method of generating functions is one of the most advanced theoretical methods of combinatorial analysis and one of the most powerful in applications. It is known that the generating function of the solution is always rational in the case of one variable and a difference equation with constant coefficients (see [3]). When passing to several variables, this is no longer the case, in particular, the generating series of the solution may be divergent.

From the point of view of enumerative combinatorial analysis, it seems expedient to split the set of solutions of a multidimensional difference equation into classes in accordance with the belonging of their generating functions to certain classes. From the standpoint of Stanley [4], the most useful and often used is the following hierarchy of classes of generating functions:

$$
\{\text { rational }\} \subset\{\text { algebraic }\} \subset\{\text { D-finite }\} .
$$

With this approach, the following problem seems natural:

If the generating function of the initial data of the Cauchy problem belongs to one of the classes of the above hierarchy, does the generating function of its solution belong to this class?

\footnotetext{
*lein@mail.ru

†t.neckrasova@gmail.com

(c) Siberian Federal University. All rights reserved
} 
If the answer to the above question is positive, then we say that the Cauchy problem preserves the Stanley hierarchy of generating functions.

The standard situation for combinatorial analysis is investigated in [1], when solutions of the difference equation are sought in the positive octant $\mathbb{Z}_{\geqslant}^{n}$ of the integer lattice $\mathbb{Z}^{n}$. In the works $[1,5]$, the preservation of the Stanley hierarchy was provided by a number of restrictions on the difference equation. The results on the preservation of the Stanley hierarchy obtained in [1] are generalized in the paper [5] to the case when solutions of the multidimensional difference equation are sought in an arbitrary simplicial rational cone $K$ of the integer lattice $\mathbb{Z}^{n}$.

In this paper, instead of restrictions on the difference equation that ensure that the Cauchy problem preserves the Stanley hierarchy, it is proposed to find a rational cone $C, C \supset K$ containing $K$ in which these conditions are fulfilled automatically and, consequently, the Cauchy problem preserves the hierarchy Stanley generating functions of the solutions of the difference equation. It is required that every solution $f$ in the cone $K$ can be obtained as a trace $f=\left.u\right|_{K}$ of some solution $u$ in the cone $C$.

This approach is realized in the first section in Theorem 1.1. In addition, it can be useful in the study of the stability of the Cauchy problem, since it allows us in some cases to find an explicit form of the fundamental solution of the Cauchy problem as the coefficients of the Laurent series expansion of the function $1 / P(z)$. Note that this played an important role in [2].

In the second section we consider the question of the connection between the generating functions of the solutions $f$ and $u$ of Cauchy problems with supports in the cones $K$ and $C$, where $K \subset C$. To answer it, the concept of the Hadamard composition of power series (see [6,7]) is generalized to Laurent series with supports in rational cones. In Proposition 2.1 we obtain an integral representation for the Hadamard composition of such series and, as a consequence, give an integral formula in which the generating function of the trace $f=\left.u\right|_{K}$ is expressed in terms of the generating function of the solution $u$ of the Cauchy problem in the rational cone $C$.

\section{The Cauchy problem and the Stanley hierarchy of generating functions of its solutions}

The Cauchy problem for a linear difference equation with constant coefficients of the form

$$
\sum_{\omega=0}^{m} c_{\omega} \delta^{\omega} f(x)=g(x)
$$

where $c_{m} \neq 0, \delta^{\omega} f(x)=f(x+\omega)$, with initial data

$$
f(k)=\varphi(k), k=0,1, \ldots, m-1,
$$

has a unique solution in an obvious way.

For $n>1$, the situation is much more complicated and the difficulties are related to the fact that the solution space of the difference equation is infinite-dimensional, and the question of additional conditions that allow to single out unique solutions in this space is non-trivial (see, for example, $[1,2,5,8])$.

It is known (see [3]) that the generating function of every solution of the Cauchy problem (1)-(2) for $n=1$ is rational, and for $n>1$ this is false and the properties of the generating function of the solution of the difference equation depend not only on the properties of the 
generating functions of the initial data, but also on the form of the equation. One way to describe this dependence is to find out the conditions under which the Stanley hierarchy is preserved.

The following notation and definitions are required for an exact formulation.

Let $a^{1}, \ldots, a^{n}$ be linearly independent vectors with integer coordinates $a^{j}=\left(a_{1}^{j}, \ldots, a_{n}^{j}\right) . A$ rational cone generated by $a^{1}, \ldots, a^{n}$, we call the set

$$
K=\left\{x \in \mathbb{R}^{n}: x=\lambda_{1} a^{1}+\cdots+\lambda_{n} a^{n}, \lambda_{j} \in \mathbb{R}_{+}, j=1, \ldots, n\right\} .
$$

Note that $K$ is a simplicial cone; i.e., each element of $K$ has the unique representation via generators.

Define on the complex-valued functions $f(x)=f\left(x_{1}, \ldots, x_{n}\right)$ of integer variables $x_{1}, \ldots, x_{n}$ the shift operators $\delta_{j}$ with respect to $x_{j}$ as

$$
\delta_{j} f(x)=f\left(x_{1}, \ldots, x_{j-1}, x_{j}+1, x_{j+1}, \ldots, x_{n}\right)
$$

and the polynomial difference operators

$$
P(\delta)=\sum_{\omega \in \Omega} c_{\omega} \delta^{\omega}
$$

where finite set of points $\Omega$ belongs to the point cone $K \cap \mathbb{Z}^{n}, \delta^{\omega}=\delta_{1}^{\omega_{1}} \cdots \cdots \delta_{n}^{\omega_{n}}$, while $c_{\omega}$ are constant coefficients of the difference operator. The functions $f(x)$ on which the operator $P(\delta)$ acts are naturally considered on the cone $K \cap \mathbb{Z}^{n}$, since it contains the set of shifts $\Omega \subset K$ and withstands shifts by $x \in K$.

Generating functions along with difference equations are one of the important tools for solving enumerative combinatorial analysis problems ( $[1,3,9-11])$. To investigate difference equations in rational cones, we require Laurent series with supports in these cones.

Refer as the generating function (series) of a function $f: K \cap \mathbb{Z}^{n} \rightarrow \mathbb{C}$ of integer arguments $x=\left(x_{1}, \ldots, x_{n}\right)$ to the Laurent series

$$
F(z)=\sum_{x \in K \cap \mathbb{Z}^{n}} f(x) z^{-x} .
$$

The series of this form (4) constitute the ring $\mathbb{C}_{K}[[z]]$ under addition and multiplication. Let us give the definition of D-finiteness for series of the form (4).

We can express each element $x \in K \cap \mathbb{Z}^{n}$ as a linear combination $x=\lambda_{1} a^{1}+\cdots+\lambda_{n} a^{n}$ of basis vectors. In matrix form this expression becomes $x=A \lambda$, where $\lambda$ is a column vector, and $A$ is the matrix with the coordinates of the vectors $a^{j}$ in columns,

$$
A=\left(\begin{array}{ccc}
a_{1}^{1} & \ldots & a_{1}^{n} \\
. . & . . & . . \\
a_{n}^{1} & \ldots & a_{n}^{n}
\end{array}\right) .
$$

The determinant $\Delta$ of $A$ is nonzero.

Define the operator $D_{i}$ on the monomials $f(x) z^{-x}$ as follows:

$$
D_{i} f(x) z^{-x}=-\lambda_{i} f(x) z^{-x-a^{i}}
$$

where $\lambda_{i}$ is the $i$ th coordinate of the point $\lambda=A^{-1} x$. 
The action of $D_{i}$ on a series $F$ in $\mathbb{C}_{K}[[z]]$ is defined by linearity. It is not difficult to verify that $D_{i}$ for $i=1, \ldots, n$ are derivations (i.e., they map $\mathbb{C}_{K}[[z]]$ into itself, are linear, and satisfy the usual Leibniz product rule for the derivative). Put

$$
D_{i}^{k}=\underbrace{D_{i} \circ \cdots \circ D_{i}}_{k \text { times }} \text {. }
$$

A formal series $F(z)=\sum_{x \in K \cap \mathbb{Z}^{n}} f(x) z^{-x}$ in the ring $\mathbb{C}_{K}[[z]]$ is called D-finite whenever it satisfies a system of equations

$$
Q_{k}^{i}(z) D_{i}^{k} F(z)+\cdots+Q_{1}^{i}(z) D_{i} F(z)+Q_{0}^{i}(z) F(z)=0, i=1, \ldots, n,
$$

with $Q_{j}^{i}(z) \in \mathbb{C}_{K}[z]$.

D-finite multiple power series were investigated in [12], and the above definition of the Dfiniteness of the Laurent series was used in [5] in connection with the study of the properties of solutions of multidimensional difference equations.

Define the partial order $\underset{K}{\geqslant}$ between the points $u, v \in \mathbb{R}^{n}$ as follows:

$$
u \underset{K}{\geqslant} v \Leftrightarrow u-v \in K
$$

Moreover, we write $u \ngtr v$ whenever $u-v \notin K$.

For fixed $m \in K \cap \mathbb{Z}^{n}$ we denote

$$
K_{m}=\left\{x \in K \cap \mathbb{Z}^{n}: \underset{K}{x \neq m}\right\}
$$

and we will call the points of this set initial (boundary) points.

We will consider difference equations of the form

$$
P(\delta) f(x)=g(x), x \in K \cap \mathbb{Z}^{n},
$$

where $f(x)$ is an unknown, and $g(x), \varphi(x)$ are given function on the set $K \cap \mathbb{Z}^{n}$. Let us state the following problem.

We need to find a function $f(x)$ satisfying the equation (7) and coinciding with a specified function $\varphi(x)$ on $K_{m}$ :

$$
f(x)=\varphi(x), x \in K_{m} .
$$

This problem is naturally called the Cauchy problem for the equation (7), and the condition (8) is the initial data of the Cauchy problem.

Conditions on the cone and the point $m$ that ensure solvability, that is, existence and uniqueness, have been studied in papers $[2,5,8,13]$.

The cone

$$
K^{*}=\left\{k \in \mathbb{R}^{n}:\langle k, x\rangle \geqslant 0, x \in K\right\},
$$

where $\langle k, x\rangle=k_{1} x_{1}+\cdots+k_{n} x_{n}$, is called dual to $K$. Denote the set of its interior points by $\stackrel{\circ}{K}^{*}$ and fix $\nu \in \stackrel{\circ}{K}^{*} \cap \mathbb{Z}^{n}$. For all $x \in K \cap \mathbb{Z}^{n}$, the nonnegative number

$$
\operatorname{deg}_{\nu} z^{x}=\langle\nu, x\rangle
$$


is referred to as the weighted homogeneous degree of $z^{x}$. The weighted homogeneous degree of the Laurent polynomial $Q(z)=\sum_{x} q_{x} z^{x}$ is defined by the formula

$$
\operatorname{deg}_{\nu} Q(z)=\max _{x \in \text { Supp } Q} \operatorname{deg}_{\nu} z^{x}
$$

Refer to the Laurent polynomial $P(z)=\sum_{\omega \in \Omega} c_{\omega} z^{\omega}$ as the characteristic polynomial of the difference operator (3).

The Newton polytope $N_{P}$ of polynomial $P(z)$ is the convex hull in $\mathbb{R}^{n}$ of the elements of $\Omega$.

Further, we will assume that $m$ is the vertex of the Newton polytope $N_{P}$ of the characteristic polynomial $P(z)$ such that $\operatorname{deg}_{\nu} P(z)=\langle\nu, m\rangle$. It follows from [5] (Theorem 1) that this condition ensures the solvability of the Cauchy problem (7)-(8).

Let $\tilde{K}$ be the cone at the vertex $m$ of the Newton polyhedron generated by the vectors $\{m-\omega, \omega \in \Omega\}$. The skeleton of a rational cone is the set of all primitive (not multiple) integer vectors in faces of dimension 1.

Instead of the problem (7)-(8) we construct another problem that will be «consistent» with problem (7)-(8) and will preserve the Stanley hierarchy. Namely, instead of the equation (7) we seek solutions of the equation

$$
P(\delta) u(x)=h(x), x \in C \cap \mathbb{Z}^{n}
$$

in the cone $C \cap \mathbb{Z}^{n}$ such that $C \supset K$ and $\left.h(x)\right|_{K}=g(x)$. It is obvious that for any solution $u(x)$ of the equation (9) the trace $\left.u(x)\right|_{K}$ is also a solution of the equation (7).

Let us denote $C_{m}=\left\{x \in C \cap \mathbb{Z}^{n}: x \underset{C}{\not} m\right\}$ and seek the solution $u(x)$ of the equation (9) which satisfies the initial data

$$
u(x)=v(x), x \in C_{m} .
$$

If in this case for each solution $f(x)$ of the problem $(7)-(8)$ there are right-hand sides $h(x)$ and initial data $v(x)$ of the problem (9)-(10) such that $f(x)=\left.u(x)\right|_{K}$, then we call the Cauchy problem (9)-(10) consistent with the problem (7)-(8).

Let us give conditions ensuring the consistency of the problem (9)-(10) and (7)-(8), as well as preserving the Stanley hierarchy.

Theorem 1.1. Let a rational, simplicial cone $C$ contains the cone $K$, and the skeleton of the cone $\tilde{K}$ contains exactly $n$ vectors. If $\tilde{K} \subset C$ and $\nu \in \stackrel{\circ}{ }^{*} \cap \mathbb{Z}^{n}$, then the Cauchy problem (9)-(10) is consistent with the problem (7)-(8) and for $h(x) \equiv 0$ the Cauchy problem (9)-(10) preserves the Stanley hierarchy of generating functions.

Proof. Let us prove the consistency of the problems. Define the function

$$
\chi_{A}(x)= \begin{cases}1, & \text { если } x \in A, \\ 0, & \text { если } x \notin A .\end{cases}
$$

We formulate the following problem:

Find the solution $u(x)$ of equation

$$
\begin{gathered}
\left.P(\delta) u(x)=g(x) \chi_{K}(x)+\sum_{\omega \in \Omega, \omega \neq m} c_{\omega} f(x+\omega)+c_{m} \varphi(x+m)\right) \chi_{\{C \cap((-m+K) \backslash K)\}}(x)+ \\
+\tilde{g}(x) \chi_{\{C \backslash(-m+K)\}}(x), x \in C \cap \mathbb{Z}^{n}, \\
-716-
\end{gathered}
$$


satisfying the initial conditions

$$
u(x)=\varphi(x) \chi_{K_{m} \cap C_{m}}(x)+\tilde{\varphi}(x) \chi_{C_{m} \backslash K_{m}}, x \in C_{m},
$$

for any functions $\tilde{\varphi}(x)$ and $\tilde{g}(x)$. This problem has a unique solution (see [2], Theorem 1) for any fixed $\tilde{\varphi}(x)$ and $\tilde{g}(x)$.

We consider the solutions $u(x)$ of the Cauchy problem (12)-(13) for arguments $x$ lying in the original cone $K$. Let $x \in K$, then

$$
\sum_{\omega \in \Omega} c_{\omega} u(x+\omega)=g(x), x \in K \cap \mathbb{Z}^{n} .
$$

If $x \in C \cap((-m+K) \backslash K)$, then

$$
u(x)=\varphi(x), x \in\left\{C \cap((-m+K) \backslash K\} \cap \mathbb{Z}^{n} .\right.
$$

Let $x \in K_{m} \cap C_{m}$, then

$$
u(x)=\varphi(x), x \in K_{m} \cap C_{m} .
$$

From (15) and (16) it follows that

$$
u(x)=\varphi(x), x \in K_{m} .
$$

Thus, $\left.u\right|_{K}$ satisfies the equation (7) and the initial data (8). By the uniqueness of the solution of the Cauchy problem, it follows that $f(x)=\left.u(x)\right|_{\left\{K \cap \mathbb{Z}^{n}\right\}}$.

Let us prove the preservation of the hierarchy. We use the following theorem from the article [5]:

If the point $m$ defining the set $C_{m}$ on which the Cauchy data of the homogeneous problem (12)-(13) are given satisfies $m \underset{C}{\geqslant} \omega$ for all $\omega \in \Omega$, then the D-finiteness (rationality or algebraicity) of the generating function of the Cauchy data implies the D-finiteness (rationality or algebraicity) of the generating function of the solution.

Note that the conditions $\tilde{K} \subset C$ and $m \underset{C}{\geqslant} \omega$ for all $\omega \in \Omega$ are equivalent. Indeed, since $\tilde{K}$ is the cone at the vertex $m$ of the Newton polyhedron generated by the vectors $\{m-\omega, \omega \in \Omega\}$, the condition $\tilde{K} \subset C$ can be rewritten as $m-\omega \in C$ for all $\omega \in \Omega$. Further, by definition, $m \geqslant \omega$ means that $m-\omega \in C$ for all $\omega \in \Omega$.

Therefore, from the above assertion on the consistency of problems and the above theorem from [5], it follows preservation of the Stanley hierarchy of generating functions of the solutions of the problem (9)-(10).

Remark 1.1. The condition $\tilde{K} \subset C$ of Theorem 1.1 is necessary for the preservation of the Stanley hierarchy, but not necessarily for the consistency of the problems (9)-(10) and (7)-(8).

\section{The Hadamard composition of Laurent series with supports in a rational cone}

The classical construction of the Hadamard composition (see [6]) of power series has many variants of generalization to multiple series that appeared in connection with the solving of some problems in number theory (see [14]) and combinatorial analysis (see [15]). One of these constructions, the "diagonal" Hadamard compositions of the double series, goes back to Poincare [16], and 
in [17] the diagonals of the double series were studied in connection with the stability problem of digital recursive filters.

Let us give a generalization of the Hadamard composition to Laurent series with supports in rational cones and an integral representation of the composition that can be useful in the following situation. We are interested in the problem of finding the generating function $F(z)$ of the solution of the problem (7)-(8) if the generating function $U(z)$ of the solution of the consistent problem (9)-(10) it is known.

We denote the cone $C=\left\{x \in \mathbb{R}^{n}: x=\lambda_{1} b^{1}+\cdots+\lambda_{n} b^{n}, \lambda_{j} \in \mathbb{R}_{+}, j=1, \ldots, n\right\}$, where $b^{1}, \ldots, b^{n}$ are linearly independent vectors with integer coordinates and

$$
\begin{aligned}
& U(z)=\sum_{x \in C \cap \mathbb{Z}^{n}} \frac{u(x)}{z^{x}}, \\
& V(z)=\sum_{x \in C \cap \mathbb{Z}^{n}} \frac{v(x)}{z^{x}}
\end{aligned}
$$

are series which supports supp $U$, supp $V$ lie in $C \cap \mathbb{Z}^{n}$, where supp $U=\left\{x \in \mathbb{Z}^{n}: u(x) \neq 0\right\}$.

The Hadamard composition of the Laurent series $U(z)$ and $V(z)$ is the series

$$
F(z)=\sum_{x \in C \cap \mathbb{Z}^{n}} \frac{v(x) u(x)}{z^{x}} .
$$

The natural domains of convergence of multiple power series are complete n-circular domains, which, by virtue of Abel's lemma (see [18]), are settled by polycylinders. For series of the form (18) the Weyl polyhedra play the role of polycylinders.

For a rational cone $C$ generated by the vectors $b^{1}, \ldots, b^{n}$, we consider a polynomial Weyl polyhedron that has the form $U_{C}^{r}=\left\{z \in \mathbb{C}^{n}:\left|z^{b^{j}}\right|>r_{j}, j=1, \ldots, n\right\}$. We formulate a version of Abel's lemma for Laurent series with supports, which lay in a rational cone.

If the series (18) converges to the point $z_{0}$, then the series (18) converges in the polyhedron $U_{C}^{r}=\left\{z \in \mathbb{C}^{n}:\left|z^{b^{j}}\right|>r_{j}, j=1, \ldots, n\right\}$ is absolutely and uniformly. This proposition is proved similarly to Lemma 7 in the paper [2].

Let us give an integral representation of the Hadamard composition for Laurent series with supports in rational cones.

Proposition 2.1. Let the functions $U(z)$ and $V(z)$ be holomorphic in the polyhedra $U_{C}^{r}=$ $=\left\{z \in \mathbb{C}^{n}:\left|z^{b^{j}}\right|>r_{j}, j=1, \ldots, n\right\}$ and $V_{C}^{R}=\left\{z \in \mathbb{C}^{n}:\left|z^{b^{j}}\right|>R_{j}, j=1, \ldots, n\right\}$ and (18), (19) are their expansions in Laurent series. Then the series (20) converges in the polyhedra $W_{C}^{R r}=\left\{z \in \mathbb{C}^{n}:\left|z^{b^{j}}\right|>R_{j} r_{j}, j=1, \ldots, n\right\}$, and for $z \in W_{C}^{R r}$, the integral representation is valid for the Hadamard composition $F(z)$

$$
F(z)=\frac{1}{(2 \pi i)^{n}} \int_{T} U(\xi) V\left(\frac{z}{\xi}\right) \frac{d \xi}{\xi}
$$

where $T=\left\{\xi \in \mathbb{C}^{n}:\left|\xi^{b^{j}}\right|=\rho_{j}, r_{j}<\rho_{j}<\frac{\left|z_{j}\right|}{R_{j}}, j=1, \ldots, n\right\}$.

Proof. It follows from the hypothesis of the theorem that for $z \in W_{C}^{R r}$ the series $U(z)$ and $V\left(\frac{z}{\xi}\right)$ converge on the skeleton $T$ absolutely and uniformly. Multiplying them and integrating term by term, we obtain:

$$
\frac{1}{(2 \pi i)^{n}} \int_{T} \sum_{x \in C \cap \mathbb{Z}^{n}} \frac{u(x)}{\xi^{x}} \sum_{y \in C \cap \mathbb{Z}^{n}} \frac{v(y) \xi^{y}}{z^{y}} \frac{d \xi}{\xi}=\frac{1}{(2 \pi i)^{n}} \int_{T} \sum_{x, y} \frac{u(x)}{\xi^{x}} \frac{v(y) \xi^{y}}{z^{y}} \frac{d \xi}{\xi}=
$$




$$
=\frac{1}{(2 \pi i)^{n}} \sum_{x, y} \int_{T} \frac{u(x)}{\xi^{x}} \frac{v(y) \xi^{y}}{z^{y}} \frac{d \xi}{\xi} .
$$

Further, using the formula $\frac{1}{(2 \pi i)^{n}} \int_{T} \xi^{k} d \xi=\left\{\begin{array}{ll}0, & \text { при } k \neq(-1, \ldots,-1), \\ 1, & \text { при } k=(-1, \ldots,-1),\end{array}\right.$ we obtain

$$
\begin{gathered}
\frac{1}{(2 \pi i)^{n}} \sum_{x, y} \int_{T} \frac{u(x)}{\xi^{x}} \frac{v(y) \xi^{y}}{z^{y}} \frac{d \xi}{\xi}=\frac{1}{(2 \pi i)^{n}} \int_{T} \sum_{x \in C \cap \mathbb{Z}^{n}} \frac{u(x) v(x)}{z^{x}} \frac{d \xi}{\xi}= \\
=\sum_{x \in C \cap \mathbb{Z}^{n}} \frac{u(x) v(x)}{z^{x}}=F(z) .
\end{gathered}
$$

Let us give an integral representation for the Hadamard composition of Laurent series and, as a consequence, give an integral formula in which the generating function of the trace $f=\left.u\right|_{K}$ is expressed in terms of the generating function of the solution $u$ of the Cauchy problem in the rational cone $C$.

We denote by $\tau=a^{1}+\cdots+a^{n}$ and $\Pi_{\tau}=\left\{x \in \mathbb{R}^{n}: 0 \underset{K}{\leqslant} \underset{K}{<} \tau\right\}$ the fundamental parallelotope associated with the set of vectors $a^{1}, \ldots, a^{n}$ (see [19]). It is shown in the paper [2] (Lemma 6) that the geometric progression $\mathcal{E}(\xi)=\sum_{x \in K \cap \mathbb{Z}^{n}} \xi^{x}$ converges for $\xi$ such that $\left|\xi^{a^{j}}\right|<1, j=1, \ldots, n$ and its sum is equal to

$$
\mathcal{E}(\xi)=\sum_{x \in K \cap \mathbb{Z}^{n}} \xi^{x}=\left(\sum_{v \in \Pi_{\tau} \cap \mathbb{Z}^{n}} \xi^{v}\right) \prod_{i=1}^{n}\left(1-\xi^{a^{i}}\right)^{-1} .
$$

We give the formula for the generating function of the trace $\left.u\right|_{K}=f$ of the solution $u$ of the problem (9)-(10). Taking in the formula (21) from Proposition 2.1 the series $V(z)=\mathcal{E}\left(\frac{1}{z}\right)=$ $=\sum_{x \in K \cap \mathbb{Z}^{n}} \frac{1}{z^{x}}=E(z)$, for $z \in W_{C}^{r}$, we obtain the integral formula

$$
F(z)=\frac{1}{(2 \pi i)^{n}} \int_{T} U(\xi) E\left(\frac{z}{\xi}\right) \frac{d \xi}{\xi}
$$

where $T=\left\{\xi \in \mathbb{C}^{n}:\left|\xi^{b^{j}}\right|=\rho_{j}, r_{j}<\rho_{j}<\left|z_{j}\right|, j=1, \ldots, n\right\}$.

Let us note that in the formula (22) the supports of the series $U$ and $E$ intersect along the cone $K$, therefore the support of $F(z)$ also lies in $K$.

We note that the special case of the formula (22) is the formula for the diagonal (see, for example, [20]). Indeed, let $C=\mathbb{Z}_{\geqslant}^{2}$ and the cone $K$ be generated by the vector $a=(p, q)$ with integer nonnegative coordinates. Taking $F(z)=\sum_{x \in K \cap \mathbb{Z}^{2}} \frac{f(x)}{z^{x}}, U(z)=\sum_{x \in C \cap \mathbb{Z}^{2}} \frac{f(x)}{z^{x}}, V\left(\frac{z}{\xi}\right)=$ $=\sum_{x \in K \cap \mathbb{Z}^{2}} \frac{\xi^{x}}{z^{x}}=\frac{1}{1-\frac{\xi^{a}}{z^{a}}}$ in the formula (21) for $z \in W_{C}^{r}$ we obtain an integral representation for the (p,q)-diagonal $U_{(p, q)}^{z^{a}}(z)$ of the series $U(z)$

$$
F(z)=U_{(p, q)}(z)=\frac{1}{(2 \pi i)^{2}} \int_{T} \frac{U(\xi)}{1-\frac{\xi^{a}}{z^{a}}} \frac{d \xi}{\xi}
$$

where $T=\left\{\xi \in \mathbb{C}^{2}:\left|\xi_{j}\right|=\rho_{j}, r_{j}<\rho_{j}<\left|z_{j}\right|, j=1, \ldots, n\right\}$. 
Let us give an example illustrating Theorem 1.1 and the formula (22) for generating functions of solutions of the Cauchy problem. We consider the two-dimensional difference equation, the appearance of which is connected with the study of combinatorial properties of root systems and their applications to Chevalley groups:

$$
f(x+1, y+1)-f(x+1, y)-f(x, y+2)=0,(x, y) \in \mathbb{Z}_{\geqslant}^{2} .
$$

For this equation $\Omega=\operatorname{supp} P(z, w)=\{(1,0),(1,1),(0,2)\}$, the cone $K=\mathbb{Z}_{\geqslant}^{2}$. Concerning the vertex $m=(1,1)$ of the Newton polyhedron we give the initial data on the set $K_{m}=\{(x, y) \in$ $K:(x, y) \underset{K}{\ngtr} m:$

$$
\begin{aligned}
& f(0, k)=0, k=0,1,2, \ldots, \\
& f(1,0)=1, \\
& f(k, 0)=0, k=2,3, \ldots
\end{aligned}
$$

Note that this type of problem was considered in the paper [21].

Since there exists a point $(0,2) \in \Omega$ such that $m \ngtr(0,2)$, then in the cone $K$ the Cauchy problem may not preserve the Stanley hierarchy (see [5]). Let us select the cone $C \supset K$ so that the condition $m \underset{K}{\geqslant} \omega$ is fulfilled, which ensures the preservation of the hierarchy. Namely, let us suppose that the cone $C$ is generated by the vectors $(1,-2)$ and $(0,1)$ and consider instead the equation (24) the difference equation

$$
u(x+1, y+1)-u(x+1, y)-u(x, y+2)=0,(x, y) \in C \cap \mathbb{Z}^{2}
$$

and the initial data on the set $C_{m}=\left\{(x, y) \in C \cap \mathbb{Z}^{2}: x \underset{C}{\ngtr} m\right\}$ :

$$
\begin{aligned}
& u(0, k)=0, \quad k=0,1,2, \ldots, \\
& u(k,-2 k)=(-1)^{k+1} k^{2}, \quad k=1,2, \ldots, \\
& u(k,-2 k+1)=(-1)^{k+1}(2 k-1), \quad k=1,2, \ldots, \\
& u(1,0)=1 \\
& u(k,-2 k+2)=(-1)^{k+1} 2, \quad k=2,3, \ldots
\end{aligned}
$$

According to the assertion of the first part of Theorem 1.1, there are initial data, such that the solution $u(x, y)$ of the new problem (26)-(27) satisfies the condition $f(x, y)=\left.u(x, y)\right|_{\left\{K \cap \mathbb{Z}^{2}\right\}}$.

According to the statement of the second part of Theorem 1.1 the Cauchy problem preserves the Stanley hierarchy for generating functions in the cone $C$. In [5] a formula for the generating function of the solution of the Cauchy problem is obtained, in which $F$ is expressed in terms of the generating functions of the initial data $\Phi_{\omega}$. We compute

$$
\begin{gathered}
\Phi_{(0,2)}=\Phi_{(1,0)}=\frac{z^{2} w^{2}-z w^{4}}{\left(z+w^{2}\right)^{3}}+\frac{3 z w^{2}+z^{2}-z-w^{2}}{w\left(z+w^{2}\right)^{2}}, \\
\Phi_{(1,1)}=\frac{z^{2} w^{2}-z w^{4}}{\left(z+w^{2}\right)^{3}}+\frac{3 z w^{2}+z^{2}-z-w^{2}}{w\left(z+w^{2}\right)^{2}}+\frac{z^{3}+z^{2} w^{2}-2 w^{2}}{z\left(z+w^{2}\right)},
\end{gathered}
$$

and using the above formula from [5], we obtain the generating function of the solution $u(x, y)$ of the problem (26)-(27)

$$
U(z, w)=\frac{z^{2} w^{2}-z w^{4}}{\left(z+w^{2}\right)^{3}}+\frac{z^{2}+3 z w^{2}-z-w^{2}}{w\left(z+w^{2}\right)^{2}}+\frac{z w}{z w-w^{2}-z}\left(z-\frac{2 z}{w^{2}\left(z+w^{2}\right)}-\frac{2}{z}+\frac{2}{w^{2}}\right) .
$$


Further, by the formula (22) we find the generating function for the solution of the problem $(24)-(25)$

$$
F(z)=\frac{2 z w-z w^{2}+w^{2} \sqrt{z(z-4)}}{2\left(z w-z-w^{2}\right)} .
$$

The first author was supported by RFFI no. 18-51-41011 Uzb_t, and the second by RFBR according to the research project no. 18-31-00232.

\section{References}

[1] M.Bousquet-Mélou, M.Petkovšek, Linear recurrences with constant coefficients: the multivariate case, Discrete Mathematics, 225(2000), 51-75.

[2] T.I.Yakovleva, Well-posedness of the Cauchy problem for multidimensional difference equations in rational cones, Siberian Mathematical Journal, 58(2017) no. 2, 363-372.

[3] R.P.Stanley, Enumerative Combinatorics, Moscow, Mir, 1990 (Russian).

[4] R.P.Stanley, Enumerative Combinatorics. Trees, generating functions, symmetric functions, Moscow, Mir, 2009 (in Russian).

[5] E.K.Leinartas, T.I.Nekrasova, Constant coefficient linear difference equations on the rational cones of the integer lattice, Siberian Mathematical Journal, 57(2016) no. 2, 98-112.

[6] L.Bieberbach, Analytic continuation, Moscow, Nauka, 1967 (in Russian).

[7] E.K.Leinartas, Hadamard's theorem on the multiplication of singularities in $\mathbb{C}^{n}$, Sib. Mat. Zh., 27(1986) no. 3, 209-212 (in Russian).

[8] E.K.Leinartas, M.S.Rogozina, Solvability of the Cauchy problem for a polynomial difference operator and monomial bases for the quotients of a polynomial ring, Siberian Mathematical Journal, 56(2015) no. 1, 111-121.

[9] A.O.Gelfond, The calculus of finite differences, Moscow, KomKniga, 2006 (in Russian).

[10] O.A. Shishkina, Bernoulli Polynomials in Several Variables and Summation of Monomials over Lattice Points of a Rational Parallelotope, Izvestiya Irkutsk Gos. Univ., 16(2016), 89-101 (in Russian).

[11] O.A.Shishkina, Multidimensional Analog of the Bernoulli polynomials and its Properties Journal of Siberian Federal University, Mathematics \& Physics, 9(2016), no. 3, 384-392.

[12] L.Lipshitz, D-Finite power series, Journal of Algebra, 122(1989), 353-373.

[13] M.S.Apanovich, E. K. Leinartas, Correctness of a Two-dimensional Cauchy Problem for a Polynomial Difference Operator with Constant Coefficients, Journal of Siberian Federal University. Mathematics \& Physics, 10(2017), no. 2, 199-205.

[14] R.W.K.Odoni, On the norms of algebraic integers, Matematica, 22(1975), 71-80.

[15] D. Ż. Djokovich, A properties of the Taylor expansion of rational function in several variables, J. of Math. Anal. and Appl., 66(1978), 679-685. 
[16] J.H.Poincare, New Methods of Celestial Mechanics, Moscow, Nauka, vol. 1, 1971 (in Russian).

[17] A.K.Tsikh, Conditions for absolute convergence of the Taylor coefficient series of a meromorphic function of two variables, Math. Sb., 182(1991), no. 11, 1588-1612.

[18] B.V.Shabat, An Introduction to Complex Analysis, Moscow, Nauka, 1969 (in Russian).

[19] J.W.S.Cassels, An Introduction to the Geometry of Numbers, Moscow, Mir, 1965 (in Russian).

[20] D.Yu.Pochekutov, Diagonals of the Laurent series of rational functions, Sib. Math. J., 50(2009), 1081-1090.

[21] T.I.Nekrasova, On the hierarchy of generating functions for solutions of multidimensional difference equations, Izvestiya Irkutsk Gos. Univ., 9(2014), 91-103 (in Russian).

\section{Задача Коши для многомерных разностных уравнений и сохранение иерархии производящих функций ее решений}

\section{Евгений К. Лейнартас}

Татьяна И. Яковлева

Институт математики и фундаментальной информатики Сибирский федеральный университет Свободный, 79, Красноярск, 660041

Россия

Для разностного уравнения с постоянными коэффициентами в рациональном точечном конусе исследуется зависимость свойств производяей функции решения задачи Коши от свойств производящей функиии начальных данных. Найдены условия, при которых производящие функиии решения остаются в тех же классах, что и производящие функции начальных данных.

Ключевые слова: многомерные разностные уравнения, задача Коши, иерархия производящих функций, композиция Адамара. 